\title{
Filigrane
}

Écoutes psychanalytiques

\section{Postface. À L'écoute de chaque analysant à nul autre pareil}

\section{Roger Dufresne}

Volume 27, numéro 2, 2018

La consultation psychanalytique aujourd'hui, entre héritages et remaniements

URI : https://id.erudit.org/iderudit/1055756ar

DOI : https://doi.org/10.7202/1055756ar

Aller au sommaire du numéro

\section{Éditeur(s)}

Revue Santé mentale au Québec

ISSN

1192-1412 (imprimé)

1911-4656 (numérique)

Découvrir la revue

Citer cet article

Dufresne, R. (2018). Postface. À L'écoute de chaque analysant à nul autre pareil Filigrane, 27(2), 139-144. https://doi.org/10.7202/1055756ar

\section{Résumé de l'article}

Se centrant sur le premier entretien et en écho aux deux fils rouges qui sous-tendent l'ensemble des articles - l'analyse constante du contre-transfert et la nécessité d'un tiers externe bien réel dans le traitement des pathologies les plus graves - l'auteur montre qu'il ne suffit pas de s'en tenir aux représentations que nous nous faisons du patient, mais qu'il importe de s'enquérir de sa conception de ses problèmes et de comment il anticipe l'aide du psychanalyste. Si la « théorie thérapeutique » du patient diffère de ce que nous pensons lui proposer, il convient de le lui expliquer brièvement et de lui permettre d'y réfléchir hors de notre présence. Le but est de lui indiquer que nous ne nous plaçons pas dans un rapport où un thérapeute prescrit un traitement à un malade, mais que nous lui offrons une relation de collaboration. Ceci permet le plus souvent d'éviter des interruptions prématurées trop fréquentes, certes décevantes pour le thérapeute, mais surtout néfastes pour les patients. 


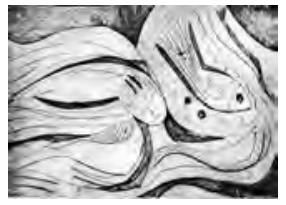

\title{
Postface. À L'écoute de chaque analysant à nul autre pareil
}

\author{
Roger Dufresne
}

\begin{abstract}
Résumé: Se centrant sur le premier entretien et en écho aux deux fils rouges qui sous-tendent l'ensemble des articles - l'analyse constante du contre-transfert et la nécessité d'un tiers externe bien réel dans le traitement des pathologies les plus graves - l'auteur montre qu'il ne suffit pas de s'en tenir aux représentations que nous nous faisons du patient, mais qu'il importe de s'enquérir de sa conception de ses problèmes et de comment il anticipe l'aide du psychanalyste. Si la «théorie thérapeutique» du patient diffère de ce que nous pensons lui proposer, il convient de le lui expliquer brièvement et de lui permettre d'y réfléchir hors de notre présence. Le but est de lui indiquer que nous ne nous plaçons pas dans un rapport où un thérapeute prescrit un traitement à un malade, mais que nous lui offrons une relation de collaboration. Ceci permet le plus souvent d'éviter des interruptions prématurées trop fréquentes, certes décevantes pour le thérapeute, mais surtout néfastes pour les patients.
\end{abstract}

Mots clés: contre-transfert; tiers externe; théorie thérapeutique du patient.

\begin{abstract}
Focussing on the first interview and reflecting the two major themes underlying all the papers - the constant analysis of counter-transference and the need for quite real external third party in the treatment of the most serious pathologies - the author shows that it is not enough to cling to the representations we have of patients, but that it is important to inquire into their conceptions of their problems and how they foresee the psychoanalyst's help. If the patients' "therapeutic theory" differs from what we are thinking about proposing to them, it is advisable to explain it to them briefly and to allow them to reflect upon it when we are not present. The goal is to show them that we are not placing ourselves in a relationship in which a therapist prescribes a treatment to a sick person, but that we are offering them a collaborative relationship. This usually makes it possible to avoid too frequent premature interruptions, which are admittedly disappointing for the therapist, but above all harmful for the patients.
\end{abstract}

Keywords: counter-transference; external third party; the patient's therapeutic theory.

e veux souligner la qualité des diverses contributions à ce recueil, toutes remarquables, qui ne se limitent pas à décrire les cadres de chaque centre 
ou clinique et les enjeux de leurs clientèles respectives, mais présentent leurs réflexions théoriques sur les choix de leurs dispositifs thérapeutiques.

Parmi les nombreux thèmes fort pertinents, la nécessité d'une tiercéité bien réelle et vivante quand un tiers interne symbolique n'a pu se constituer et que le cadre, essentiel pour l'analyste autant que pour l'analysant, s'avère insuffisant à endiguer la violence d'une relation trop exclusivement duelle, que ce tiers se nomme directeur, analyste répondant ou analyste évaluateur distinct de l'analyste traitant, auquel le patient peut se référer lors d'une crise ou d'une impasse. Je souligne qu'un tiers externe vaut aussi bien pour les thérapeutes des cas difficiles que pour les rencontres groupales d'évaluations ou de suivis cliniques. En vérité, un analyste ne peut exister seul. À un moment ou un autre, il a besoin de prendre quelque recul et de se référer à un collègue pour quelques échanges ou à un séminaire d'intervision clinique afin de surmonter des affects trop intenses ou quelque blocage ou point aveugle, en respectant la confidentialité' de ses analysants.

Alors que l'on parle d'une crise de la psychanalyse avec diminution des demandes de cures-types et de formation, je ne peux que me réjouir de voir tous les efforts qui visent à étendre le champ du psychanalytique. Le temps me paraît dépassé où l'on ne considérait analytique que la cure-type à au moins quatre séances par semaine. Les textes ici rassemblés nous montrent clairement combien et comment les analystes peuvent aider les patients les plus fragiles et les plus démunis. Ceci ne diminue en rien la pertinence de la cure-type lorsqu'indiqué et possible et pour la formation du psychanalyste et du psychothérapeute. Par contre, si l'on considère les pathologies plus graves où prédominent l'effervescence pulsionnelle de leur monde intérieur, les processus primaires et les tendances aux passages à l'acte ou aux troubles de la pensée, une écoute plus active et une présence plus manifeste s'avèrent essentielles tout en conservant une perspective psychanalytique.

Je voudrais partager avec vous quelques réflexions que m’a inspirées le thème mis au travail, d'autant plus qu'il porte sur la rencontre inaugurale à toute intervention thérapeutique, un thème qui a occupé une place centrale dans mon enseignement, car le premier entretien est l'unique occasion pour des étudiants d'observer l'interaction d'un psychanalyste avec un patient.

Le sous-titre «Entre héritage et remaniements» m’a d'abord conduit à me remémorer mes propres héritages. Quand j'ai commencé ma formation 
psychiatrique aux États-Unis à la Menninger School of Psychiatry, la grande école de l'époque, avec 150 médecins résidents en psychiatrie (l'équivalent des internes des hôpitaux en France), on nous enseignait à faire des études de cas extrêmement détaillées qui nécessitaient quatre, cinq ou six entretiens préliminaires. Dans nos rapports, il fallait y inclure les observations de la travailleuse sociale qui avait interviewé tous les membres de la famille et les conclusions du psychologue qui avait administré une large batterie de tests. Comme ce centre était d'oriention très psychanalytique, on nous demandait d'ajouter à l'examen mental psychiatrique classique des observations sur le surmoi, l'idéal du moi, l'estime de soi, les mécanismes de défense, la force du moi, l'insight, la capacité d'introspection, etc., et, pour chacun, un paragraphe pour justifier notre évaluation, sans compter une formulation psychodynamique, un diagnostic différentiel, un pronostic et des recommandations pour le traitement. Ceci était très formateur, mais présentait le grand inconvénient que, lorsque nous entreprenions une psychothérapie, le patient s'attendait à ce que nous poursuivions sur ce mode directif et était complètement désemparé quand nous l'invitions à associer librement et demeurions silencieux.

Lorsqu'ensuite je me suis rendu à Paris pour ma formation à l'Institut de psychanalyse où $j$ 'ai fait mes trois cures de contrôle au Centre de consultations et de traitements psychanalytiques - qui ne s'appelait pas encore Jean-Favreau, car celui-ci en était encore le directeur; un homme accueillant, chaleureux et fin clinicien qui fut l'un de mes superviseurs -, on nous enseignait exactement l'inverse. On nous expliquait que pour ne pas compromettre le transfert à venir, le futur analyste devait s'abstenir de faire une évaluation, si celle-ci avait déjà été faite par un analyste référant, et que lors de notre première rencontre avec l'analysant, il convenait de nous limiter à nous entendre avec lui sur les horaires des séances. J'ai donc choisi mon premier cas de contrôle sur un dossier très bref qui portait la mention: «Patiente de structure hystéro-phobique. Bon cas pour un débutant». C'était exactement ce qu'il me fallait. Or, quelques semaines après le début de l'analyse, la jeune analysante s'est mise à halluciner son dernier amant aplati comme un tapis persan volant derrière la fenêtre derrière nous. Il y eut d'autres épisodes hallucinatoires et délirants. J'ai pensé qu'il aurait mieux valu faire une évaluation plus poussée, mais je n'ai pas osé à l'époque en faire part à mes professeurs, sans doute pour ne pas être qualifié d'obsessionnel ou d'américain. Je suis heureux que les choses aient été remaniées depuis lors. 
Malgré leurs inconvénients, ces deux modèles, mes deux principaux héritages, m'ont appris deux choses essentielles: l'importance d'une bonne évaluation et celle de ne pas laisser transparaitre trop de nous-mêmes. Ainsi, au début de ma pratique, et ce fut mon premier remaniement, je me suis limité à demander aux patients ce qui les amenait et, si nécessaire, de poser une ou deux questions très générales sur leur famille ou leur enfance. Si cette approche convenait bien à des organisations névrotiques, je découvris rapidement que cela n'était guère suffisant avec des personnalités-limites ou pré-psychotiques ou de grands somatisants. J'ai donc dû remanier peu à peu mon premier remaniement et intervenir un peu plus souvent de façon plus soutenante avec certains, tout en évitant d'être trop précis ou directif, selon ce que je percevais de l'angoisse ou de la fragilité de chacun.

L'autre question fort importante que j'évoquerai brièvement concerne les suites du premier entretien. Depuis longtemps, je suis préoccupé par le fait que souvent, trop souvent, des patients interrompent leur psychothérapie ou leur psychanalyse prématurément après quelques mois, au grand désarroi du thérapeute ou de l'analyste qui s'y était beaucoup investi. Je ne connais pas de livres ou d'articles qui traitent de ce problème, sinon diverses statistiques ici et là qui rapportent de 30 à $40 \%$ d'interruptions après trois mois seulement. J'ai eu l'occasion à diverses reprises et dans divers contextes de voir en consultations certains d'entre eux ou d'interroger mes propres analysants qui m’annonçaient leur départ imminent. Je précise immédiatement que ceci ne concerne pas que les jeunes thérapeutes ou les analystes en formation, mais nous tous à des degrés divers. Il y a certes plusieurs causes à ces interruptions précoces: les résistances, l'angoisse de revivre des affects trop douloureux, un transfert d'emblée très négatif, plus rarement quelque «agir contre-transférentiel. Le plus souvent, toutefois, il m’a semblé qu'il $\mathrm{y}$ avait eu dès le départ un grave malentendu entre les deux membres du couple analytique. En effet, ce que nous entendons par psychothérapie et psychanalyse n'a pas nécessairement la même signification pour tous les patients. Certains croient qu'après quelques mois nous leur dirons de quoi ils souffrent et ce qu'ils devraient faire. Tel autre se représentait la psychanalyse comme un examen de laboratoire au terme duquel je lui remettrais un rapport. Aussi m'est-il apparu peu à peu nécessaire à la fin d'un premier entretien de m'enquérir de leurs représentions des sources de leurs 
difficultés et de l'aide qu'ils attendent de nous, ce que j'ai déjà dénommé leur "théorie étiologique" et leur "théorie thérapeutique". Souvent, on nous répond que c'est nous qui sommes les spécialistes. Si nous insistons qu'ils en ont vraisemblablement entendu parler par des amis ou dans une revue ou à la télévision, il est exceptionnel qu'ils ne nous confient pas leur vision et leurs attentes. S’ils ont déjà entrepris une psychothérapie, quelle qu'elle soit, je les invite à m'en dire le contexte et le déroulement. Ce fut pour moi un autre remaniement. Je n'ai guère trouvé de textes qui abordent ce sujet, sauf certains qui recommandent de s'assurer de la motivation des patients. Or, beaucoup de ceux-ci se disent très motivés, en espérant une prise en charge rapide, sans comprendre ce qu'implique vraiment une psychothérapie psychanalytique ou une psychanalyse.

Une investigation plus précise qui ne prend que quelques minutes me parait présenter plusieurs avantages. Tout d'abord, elle indique aux patients que nous ne faisons pas que les examiner tel un médecin avant de leur prescrire un traitement conforme à un protocole pré-établi, mais que nous souhaitons tenir compte de leur opinion. D'autre part, cette même approche nous permet de mieux leur présenter en quoi consiste ce que nous leurs proposons. Cela évite de nous engager sur la base d'un malentendu dans un processus voué à l'échec, occurrence toujours pénible pour les thérapeutes, mais plus grave encore pour les patients qui n'oseront plus entreprendre une autre psychothérapie. Aussi, quand l'écart est très grand entre la demande du patient et notre offre qui est une demande d'une demande, il convient, après lui avoir expliqué ce que nous lui offrons, d'éviter toute précipitation, quel que puisse en être notre désir. Il est préférable de surseoir à tout accord immédiat de sa part et de lui suggérer d'y réfléchir hors de notre présence et de nous rappeler éventuellement, afin qu'il soit très clair que la décision est la sienne. Une telle retenue, aussi bien en milieu hospitalier qu'en pratique privée, permet d'épargner bien des déceptions à tous, analysants et analystes, patients et thérapeutes. Beaucoup ont rappelé dans la quinzaine, certains plus tard, l'un après dix ans. J'en ai invité plus d'un à venir m'en reparler sans s'engager s'ils le désiraient.

Pour terminer, je dirai simplement: "Poursuivons toujours notre recherche, nos réflexions, nos remises en question de nos héritages et de nos propres remaniements, et l'analyse de nos transferts et contre-transferts. 
144 Filigrane, 2018

Quelles que soient nos théories cliniques, privilégions avant tout une écoute attentive et souple de chaque analysant à nul autre pareil. Ne concluons pas.»

Roger Dufresne roger.dufresne@icloud.com 